\title{
Isolation and identification of canine adenovirus type 2 from a naturally infected dog in Korea
}

\author{
Dong-Kun Yang*, Ha-Hyun Kim, Soon-Seek Yoon, Hyunkyoung Lee, In-Soo Cho \\ Viral Disease Research Division, Animal and Plant Quarantine Agency, Ministry of Agriculture, Food and Rural Affairs, \\ Gimcheon 39660, Korea
}

(Received: August 13, 2018; Revised: November 29, 2018; Accepted: December 5, 2018)

\begin{abstract}
Canine adenovirus type $2(\mathrm{CAV}-2)$ infection results in significant respiratory illness in dogs. Isolating and culturing CAV-2 allows for investigations into its pathogenesis and the development of vaccines and diagnostic assays. In this study, we successfully isolated a virus from a naturally infected dog in Gyeonggi-do, Korea. The virus was propagated in Madin-Darby canine kidney (MDCK) and Vero cells and showed a specific cytopathic morphology that appeared similar to a bunch of grapes. The virus was first confirmed as CAV-2 based on these cytopathic effects, an immunofluorescence assay, hemagglutination assay, and electron microscopy. The viral titer of the isolate designated APQA1601 reached $10^{6.5} 50 \%$ tissue culture infections dose per $\mathrm{mL}$ in MDCK cells and exhibited no hemagglutination units with erythrocytes from guinea pig. The virus was also confirmed by polymerase chain reaction and next-generation sequencing. The APQA1601 strain had the highest similarity ( 99.9\%) with the Toronto A26/61 strain, which was isolated in Canada in 1976 when the nucleotide sequences of the full genome of the APQA1601 strain were compared with those of other CAV strains. Isolating CAV-2 will help elucidate the biological properties of CAV-2 circulating in Korean dogs.
\end{abstract}

Keywords: canine adenovirus type 2, dogs, virus isolaltion

\section{Introduction}

Two different types of canine adenoviruses (CAVs) have been identified by hemagglutination (HA) and neutralization tests, as well as genetic analyses [9]. CAV belongs to the genus Mastadenovirus (family Adenoviridae) and consists of a non-enveloped, double-stranded DNA genome of approximately $32 \mathrm{~kb}$ with short inverted terminal repeats containing the origin of DNA replication. CAV type 1 (CAV-1) causes infectious canine hepatitis, whereas CAV-2 has an affinity for the respiratory tract epithelium and induces infectious laryngotracheitis, also known as kennel cough $[17,18]$. One study indicated that dogs infected with CAV-2 may have enteric issues [12]. CAV-1 and -2 infections have been reported in several species, such as dogs, wolves, coyotes, skunks, foxes, bears, and lions worldwide $[5,8,19]$. CAV is transmitted by direct contact with saliva, feces, urine, and respiratory secretions of infected individuals [3].

Diagnoses of CAV infections are usually based on virus isolation, pathological findings, polymerase chain reaction (PCR), and serological assay [2]. In Korea, CAV-1 infections have been reported in a Eurasian river otter with prolonged anorexia and weight loss [13] and in a fennec fox [4]. Some cases of CAV-2 infections in stray dogs have been detected [20]. Among the various diagnostic methods, isolation of CAV-2 allows evaluation of the efficacy of CAV vaccines and development of serologic diagnostic assays using CAV. Although CAV-2 can be isolated from several kinds of cells, there are no reports regarding successful culture of CAV-2 isolates in Korea. However, many studies have isolated CAV2 from dogs since CAV was first identified [1, 3].

CAV has three main structural proteins: the hexon, fiber, and penton base. Among these, the fiber protein attaches to the viral capsid and interacts with the cellular coxsackievirus and adenovirus receptor [10]. The early CAV genes are expressed prior to replication of the viral genome, and the late genes are expressed after replication [16]. Four regions of early-phase transcription have been identified (E1-E4). The E3 protein interacts with components of the immune system and plays important roles in pathogenesis [9]. Analysis of the structural proteins within CAV has enabled identification of the epidemiological and molecular statuses of CAV-2 isolates.

In this study, we isolated a field strain of CAV-2 from a naturally infected dog using Madin-Darby canine kidney (MDCK) and Vero cells. A CAV-2 isolate designated as APQA1601 was propagated successfully, and the APQA1601

*Corresponding author

Tel: +82-54-912-0785, Fax: +82-54-912-0812

E-mail: yangdk@korea.kr 
strain was passaged 10 times in continuous cell line. We investigated the biological properties of the APQA1601 strain after serial passage, and the nucleotide sequence of the APQA1601 strain was determined to evaluate genetic relationships. This study will enhance our understanding of CAV-2 circulating in Korean dogs.

\section{Materials and Methods}

\section{Samples}

Two month old female dog who lived in a house in Seoul, died of respiratory illness and was transferred to the Animal and Plant Quarantine Agency (APQA) for diagnosis of the disease in 2016. After necropsy, the lung, liver, brain, and small intestine tissues were collected and used to prepare a $10 \%$ homogenate in Dulbecco's modified Eagle's medium (DMEM). Following centrifugation at $4,000 \times \mathrm{g}$ for $15 \mathrm{~min}$ at $4^{\circ} \mathrm{C}$, the supernatant was filtered through a $0.45-\mu \mathrm{m}$ pore-size syringe filter (Millipore, USA) and used to isolate the virus.

\section{Virus isolation and titration}

The MDCK (CCL-34; ATCC, USA) and Vero cells (ATCC CCL-1586) used to isolate the virus were cultured in DMEM containing two antibiotics at a concentration of $100 \mathrm{IU} / \mathrm{mL}$ penicillin, $10 \mu \mathrm{g} / \mathrm{mL}$ streptomycin, and antifungal agent at a concentration of $0.25 \mu \mathrm{g} / \mathrm{mL}$ amphotericin B with $10 \%$ heatinactivated fetal bovine serum (FBS; Gibco-BRL, USA). Two kinds of cells planted in six-well plates were incubated at $37^{\circ} \mathrm{C}$ in a $5 \% \mathrm{CO}_{2}$ incubator. Confluent MDCK and Vero cells washed twice with phosphate-buffered saline (PBS; $\mathrm{pH}$ 7.2) were inoculated with $200 \mu \mathrm{L}$ of the final samples and incubated under the conditions mentioned above for $2 \mathrm{~h}$. After incubation, the inoculated samples were removed, and $5 \mathrm{~mL}$ fresh DMEM containing 5\% FBS were added to each well. The plates were maintained at $37^{\circ} \mathrm{C}$ in a $5 \% \mathrm{CO}_{2}$ incubator for 10 days. When specific cytopathic effects (CPEs) were observed in cells of the plate, the cell supernatants were collected for further passage and inoculated into freshly prepared cells again. If no clear CPEs were observed 10 days after harvesting the cell supernatant, the two cell types were fixed in $80 \%$ cold acetone and subjected to an indirect immunofluorescence assay (IFA). If CPEs and IFA results were negative after the second passage, the sample was considered negative for the virus isolation. The viral titer of isolate was carried out according to the Reed and Muench method [14]. In brief, 10-fold dilution series of the isolate were prepared in $5 \mathrm{~mL}$ tube and each 10-fold dilution was distributed into 96-well microplates and $100 \mu \mathrm{L}$ of cells were added to each well at a concentration of 20,000 cells. After incubation, CPEs were observed under a microscope daily for 6 days. As the infected cells exhibit a clear CPE, the viral titers were measured by the $50 \%$ endpoint.

\section{IFA}

The Vero cells infected with the APQA1601 strain in 24- or 96-well plates were fixed in $-20^{\circ} \mathrm{C}$ freezer with $80 \%$ acetone for $20 \mathrm{~min}$. After washing them three times with PBS ( $\mathrm{pH}$ 7.2), the fixed cells were reacted with standard diagnostic agent such as a mouse monoclonal antibody against CAV1 or CAV-2 (VMRD, USA) at $37^{\circ} \mathrm{C}$ for $45 \mathrm{~min}$ and then stained with a $100 \times$ dilution of FITC-conjugated goat-anti-mouse IgG + IgM antibodies (KPL Laboratories, USA). After washing the plates with PBS, the stained Vero cells were examined under a fluorescence microscope in various magnification ranges (Eclipse TE2000-U; Nikon, Japan). Samples showing specific fluorescence in the nucleus of the cells were considered positive for CAV-1 and 2.

\section{Electron microscopy (EM)}

The Vero cells infected with APQA1601 were collected using a rubber policeman at 4 days post-infection (dpi). After centrifuging the cells, $2.5 \%$ glutaraldehyde solution in PBS for high resolution was used as first fixative of the cell pellet and $1 \%$ osmium tetroxide solution in PBS was used as the second fixative for $2 \mathrm{~h}$. After dehydration, the fixed cells were embedded in resin, and ultrathin sections of the cells were prepared with microtome for staining. The sections were stained with both uranyl acetate and lead citrate and viral particles in the sections were observed under an electron microscope (H-7100FA; Hitachi, Japan).

\section{HA assay}

The CAV-1 Bo213 strain, which has been used as the CAV1 vaccine strain for dogs in Korea, and the APQA1601 strain were propagated in Vero cells and subjected to HA assay. The HA assay was performed at $4^{\circ} \mathrm{C}$ by preparing serial twofold dilutions of APQA1601 and Bo213 in $50 \mu \mathrm{L}$ PBS (pH 7.2) and incubating with $50 \mu \mathrm{L} 0.6 \%$ erythrocytes obtained from pigs, guinea pigs, fowl, and mice. The HA titer was expressed as the reciprocal of the highest dilutions of $\mathrm{CAV}-1$ and 2 that showed a HA reaction.

\section{PCR}

Viral DNA was extracted from the APQA1601 isolate using the Viral Gene-spin Viral DNA/RNA Extraction Kit (iNtRON Biotechnology, Korea), in accordance with the manufacturer's instructions. The extracted DNA was eluted in $50 \mu \mathrm{L}$ RNase- and DNase-free water. PCR was carried out using three kinds of specific primer sets to amplify the CAV $\mathrm{F}$ gene (Table 1). Each reaction is composed of PCR premix (Bioneer, Korea) including $10 \mu \mathrm{L}$ denatured DNA, $1 \mu \mathrm{L}$ each primer $(50 \mathrm{pmol})$, and $38 \mu \mathrm{L}$ distilled water in a $50 \mu \mathrm{L}$ total volume. After the first denaturation at $95^{\circ} \mathrm{C}$ for $5 \mathrm{~min}$, the profile proceeded to the cycle of denaturation-annealing-extension. Each temperature and time was at $95^{\circ} \mathrm{C}$ for $30 \mathrm{sec}$, at $50^{\circ} \mathrm{C}$ for $30 \mathrm{sec}$, at $72^{\circ} \mathrm{C}$ for $1 \mathrm{~min}$, and a final extension was at $72^{\circ} \mathrm{C}$ for $5 \mathrm{~min}$. The PCR products were loaded onto $2.0 \%$ agarose gel containing the RedSafe Nucleic Acid Staining Solution (iNtRON Biotechnology) and the expected sizes were checked after electrophoresis. 
Table 1. List of primers used for polymerase chain reaction analysis of canine adenovirus (CAV) type 2

\begin{tabular}{|c|c|c|c|}
\hline Primer & Oligonucleotide (3'-5') & Size (bp) & Gene region \\
\hline CAV2F1 & TCCCGTTCACCAGCACCAGGGCC & \multirow{2}{*}{757} & \multirow{2}{*}{ Fiber } \\
\hline CAV2R1 & GGTGAGAGGCGGGGGAGGGGT & & \\
\hline CAV2F2 & TGCCGGCCTTGCCGTGCAGA & \multirow{2}{*}{549} & \multirow{2}{*}{ Fiber } \\
\hline CAV2R2 & TCGATACCCCCCCTCTCCCA & & \\
\hline CAV2F3 & CCAACCCCCAGCTGCCCCCA & \multirow{2}{*}{630} & \multirow{2}{*}{ Fiber } \\
\hline CAV2R3 & CCTGAAGGCGGCAGCCTTACC & & \\
\hline
\end{tabular}

F, forward; R, reverse.

APQA1601

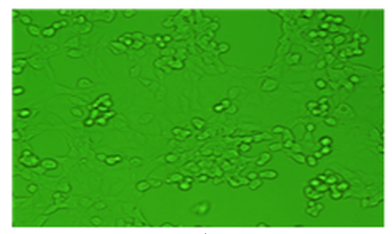

A

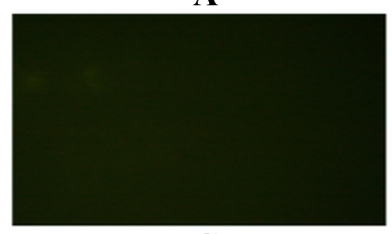

C

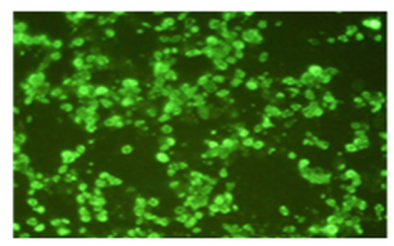

E
Normal Vero cell

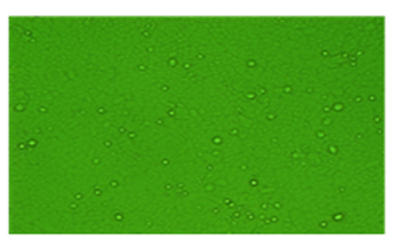

B

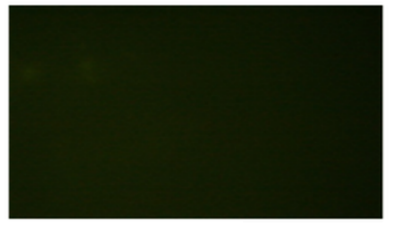

D

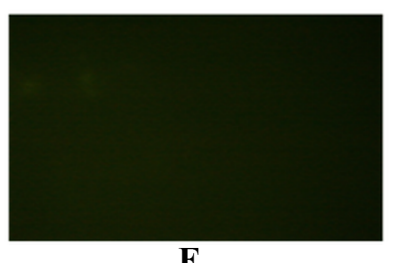

F

Fig. 1. Cytopathic effects (CPEs) of CAV type 2 (CAV-2) isolates in infected Vero cells (A) and normal Vero cells (B). Indirect immunofluorescence assay using monoclonal antibodies against CAV-1 (C and D) and CAV-2 (E and F). Vero cells infected with the APQA1601 isolate showed specific CPEs, and intranuclear fluorescence (E) was observed in Vero cells stained with the CAV-2 antibody. $200 \times(\mathrm{A}-\mathrm{F})$.

\section{Nucleotide sequencing and phylogenetic analyses}

Viral DNA extracted from the APQA1601 strain was sent to an external agency (Macrogen, Korea) to confirm the complete genome sequence using a next-generation sequencing (NGS) technique. The whole genome sequence was compared with those of the CAV reference strains. In addition, a phylogenetic analysis was performed based on the fiber genes of the CAV-2 isolate and nine other CAV strains obtained from the GenBank database. Multiple DNA sequence alignments and nucleotide similarities were examined using Clone Manager Basic ver. 9 software (Sci-Ed Software, USA). A phylogenic tree was constructed by applying the neighborjoining method using MEGA7.0.14 software [7]. The bootstrap method with 1,000 replicates was constructed to verify the reliability of the phylogenetic tree.

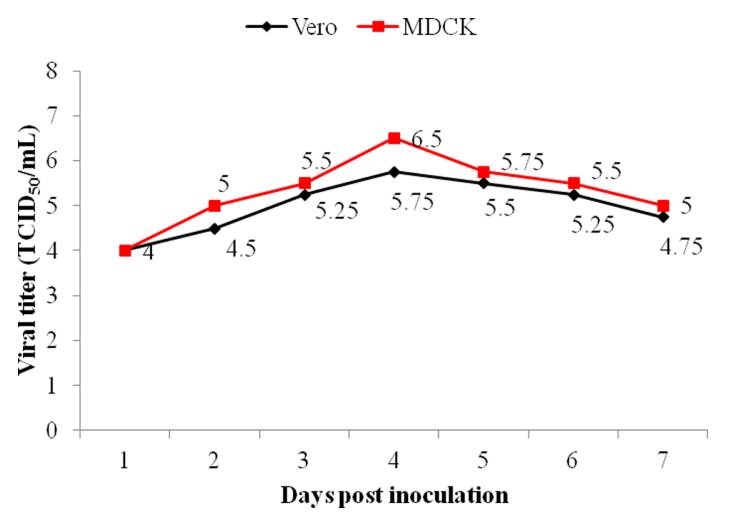

Fig. 2. Growth curves of the APQA1601 strain at passage 10 according to the time of harvest from Vero and Madin-Darby canine kidney (MDCK) cells. The APQA1601 strain showed higher proliferation in MDCK cells than in Vero cells.

\section{Results}

\section{Biological characterization and identification of CAV-2}

The virus was isolated from Vero and MDCK cells inoculated with a $10 \%$ homogenate of tissues from several organs of a CAV-infected dog. A virus showing CPEs grew in both cell types inoculated with lung and liver samples. As shown in Figure 1A, CPEs, defined as cell rounding and detachment, were observed and designated as the APQA1601 strain after the second passage. The isolate was continually passaged to obtain sufficient quantities and to confirm an accurate identification. Distinct CPEs were continually observed in the Vero and MDCK cells infected with the isolate within $5 \mathrm{dpi}$. After fixing the cells in cold acetone and staining with monoclonal antibodies against $\mathrm{CAV}-1$ and $\mathrm{CAV}-2$, respectively, specific fluorescence was detected in the nucleus of Vero cells stained with the CAV-2 monoclonal antibody (Fig. 1E). The APQA1601 strain was serially passaged 10 times in Vero and MDCK cells, and the viral titers of passage-10 APQA1601 propagated in the same cells grown in $25 \mathrm{~cm}^{2}$ culture flasks peaked at $4 \mathrm{dpi}$, reaching $10^{5.75}$ and $10^{6.5} 50 \%$ tissue cells infections dose per $\mathrm{mL}\left(\mathrm{TCID}_{50} / \mathrm{mL}\right)$ (Fig. 2). The HA assay was performed using several kinds of erythrocytes. As shown in Table 2, the isolate did not agglutinate with erythrocytes of four kinds of animals. Many CAV-2 particles in Vero cells inoculated with the APQA1601 strain were 
Table 2. Hemagglutination activity of CAV isolates using red blood cells from several species

\begin{tabular}{cccc}
\hline \hline \multirow{2}{*}{ Species } & \multicolumn{3}{c}{ Hemagglutination activity of the CAVs } \\
\cline { 2 - 4 } & APQA1601 & Bo213 (CAV-1) & Conditions \\
\hline Pig & $<2$ & $<2$ & $4^{\circ} \mathrm{C}, 0.6 \%$ Red blood cells \\
Guinea pig & $<2$ & 128 & in phosphate-buffered saline \\
Fowl & $<2$ & 64 & $(\mathrm{pH} 7.2)$ \\
Mouse & $<2$ & $<2$ & \\
\hline
\end{tabular}
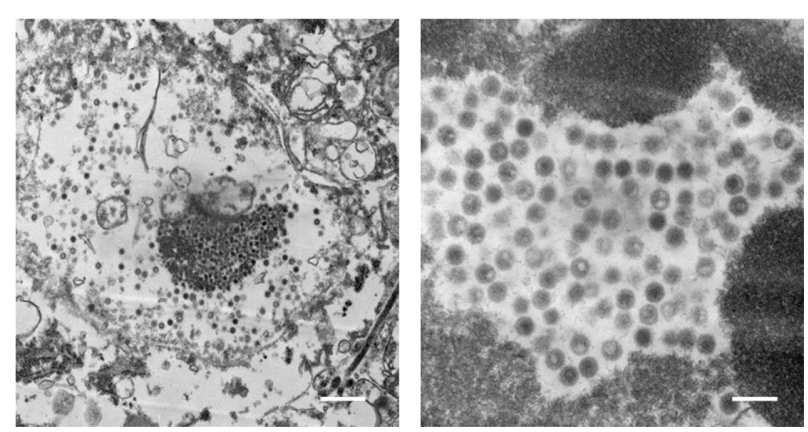

Fig. 3. Viral particles from the APQA1601 strain propagated in Vero cells. CAV-2 particles of $60-80 \mathrm{~nm}$ in diameter are visible in the nucleus and cytoplasm. Scale bars $=500 \mathrm{~nm}(\mathrm{~A}), 100 \mathrm{~nm}$ (B).

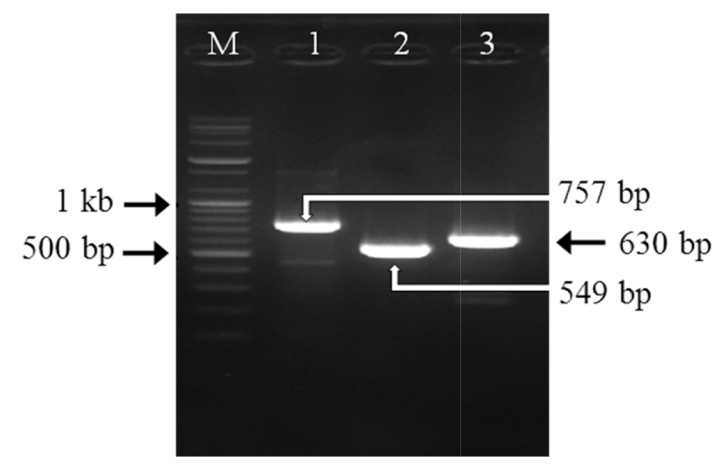

Fig. 4. Three primer sets targeting the F gene of the APQA1601 isolate were used for polymerase chain reaction (PCR). PCR products of the expected sizes confirmed the identification of the isolate as CAV-2.

A

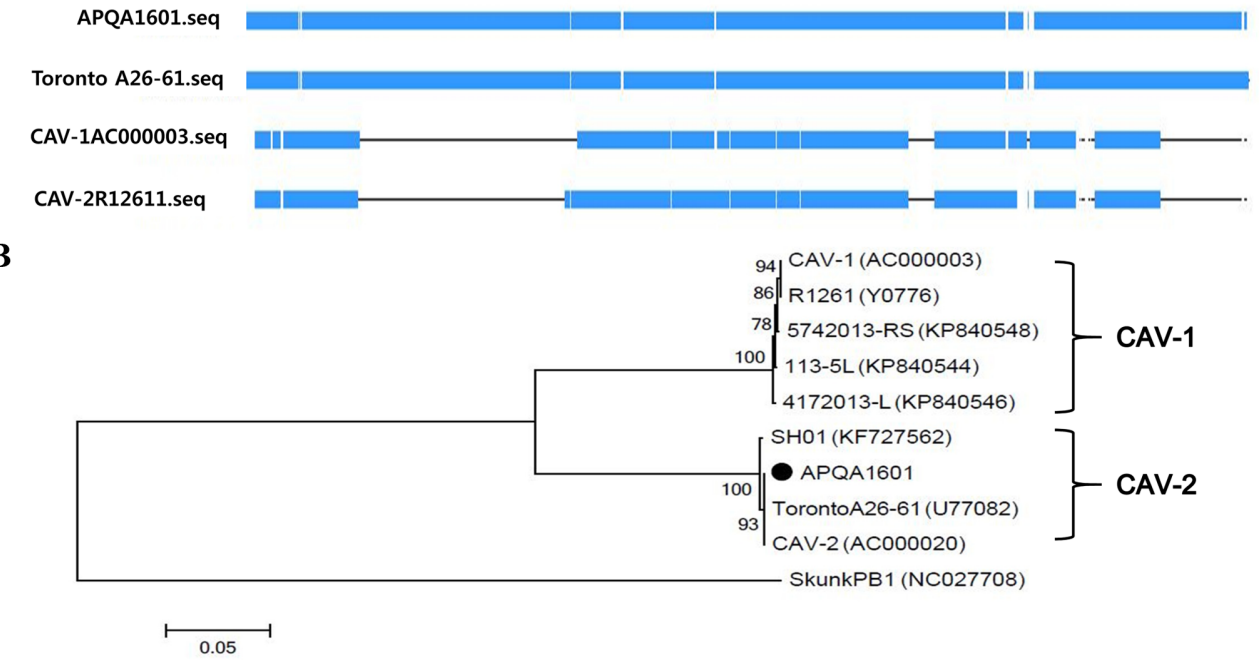

Fig. 5. Comparison of the full nucleotide sequences among four CAVs (A). A phylogenetic analysis based on the nucleotide sequences of the fiber genes from 11 adenoviral strains (B). The APQA1601 strain had the highest homology with the Toronto A26/61 strain isolated in Canada. The phylogenetic tree was constructed based on alignments of nucleotide sequences obtained using the neighborjoining method.

observed by EM. As shown in Figure 3, the viral particles were $60-80 \mathrm{~nm}$ in diameter with penton capsomeres, indicating a morphological appearance of typical Adenoviridae.

Molecular characterization and identification of CAV-2

Molecular methods were also applied to confirm the identification of CAV-2. Three partial F genes in the APQA1601 strain were amplified by PCR using specific primers for CAV-2. As shown in Figure 4, three F gene PCR products from the APQA1601 strain were detected at sizes of 757 , 630 , and 549 bp after $2.0 \%$ agarose gel electrophoresis. The complete nucleotide sequence of the APQA1601 strain was determined by NGS. The DNA genome of the APQA1601 stain was 31,093 nucleotides in length and consisted of 29 
open reading frames. The entire APQA1601 genome sequence was compared with those of one CAV-2 and two CAV-1 strains available in GenBank to determine how its genetic relationship with other CAV strains. The APQA1601 strain showed the highest nucleotide homology with the CAV-2 Toronto A26/61 strain isolated in Canada in 1976 (Fig. 5A). The E3 and F genes of the APQA1601 strain showed 100\% homology with the Toronto A26/61 strain. A phylogenetic tree was constructed based on the $\mathrm{F}$ genes of nine CAV strains to better understand the genetic relationships of the isolate. As shown in Figure 5B, CAV was divided into two clades, and the APQA1601 strain was classified as CAV-2.

\section{Discussion}

CAV infections in dogs have been reported in many countries including Korea $[3,4,13,18]$. Dogs infected with field CAV strain may die if they are not immunized with a canine combination vaccine that includes the CAV antigen [6]. CAV1 and CAV-2 infections have been identified in wild animals, such as red fox $[11,19]$. Even though diagnosing a CAV-2 infection is not difficult compared with other diseases, there have been few reports on CAV-2 isolation from Korean dogs. Thirty-eight cases have been confirmed as CAV-related infections in dogs commissioned to the APQA since 2000 in Korea (data not shown). As a dead dog was commissioned to the APQA for diagnosis in 2016, an attempt was made to isolate the causative agent from the dead dog and feces. We isolated one causative organism from lung and liver homogenates of the dog, propagated it in Vero and MDCK cells, and designated it as the APQA1601 strain. It was confirmed to be CAV-2 based on CPEs, IFA, HA assay, EM, PCR, and NGS results.

In this study, we found that MDCK cells were more suitable for isolating and propagating the field CAV-2 strain than were Vero cells based on the viral titer. Although the APQA1601-10P viral titer reached $10^{6.5} \mathrm{TCID}_{50} / \mathrm{mL}$ in MDCK cells at $4 \mathrm{dpi}$, this was slightly lower than the $10^{8.0} \mathrm{TCID}_{50} /$ $\mathrm{mL}$ of the OD-N strain [17], indicating that the APQA1601 strain was not yet fully adapted to the MDCK cells, and that continuous passage of the APQA1601 strain is required for adaptation. The APQA1601 strain showed no agglutination with red blood cells from four kinds of animals. In contrast, the CAV-1 Bo213 strain, showed agglutination with red blood cells from guinea pig, and fowl, suggesting that the HA activity of CAV-1 allows measurements of HA inhibition antibodies against CAV-1 in animals. The HA activity of the APQA1601 strain was similar to that of the OD-N strain isolated from a dog with a mild respiratory disease in Japan [17].

When the full genome sequences of CAV registered in NCBI were compared with that of the APQA1601 strain, the nucleotide sequence of the APQA1601 strain was similar to that of the Toronto A26/61strain, except for nucleotide deletions. Although double-stranded DNA viruses including CAV have lower mutation rates than those of single-stranded DNA viruses [15], the APQA1601 showed very high similarity with the Toronto A26/61 strain. Phylogenetic analyses based on the $\mathrm{F}$ gene of nine $\mathrm{CAV}$ strains revealed that the APQA1601 strain had the closest relationship with the Toronto A26/61 strain isolated in 1976. Although the Toronto A26/61 strain showed the highest homology with the APQA1601 strain, it remains unclear how CAV-2 was introduced in Korea, as the sequence information of CAV-2 in GenBank is very limited. Although CAV-2 infection has been effectively controlled in Korea by vaccination, confirmation of CAV-2 in a dead dog means that CAV-2 is still circulating in non-immunized dogs. Further studies are needed to investigate the pathogenicity of the APQA1601 strain in dogs

In conclusion, we isolated an adenovirus from a naturally infected dog and confirmed it to be CAV-2 based on a biophysical and molecular characterization. After adapting the APQA1601 strain to MDCK cells after serial passages, we determined its complete nucleotide sequence by NGS and then analyzed the obtained sequence, including the E3 and F genes. The isolate was closely related genetically to the Toronto A26/61 strain. In addition, the APQA1601 strain will be useful as an antigen for serological testing or as a challenge strain to examine the efficacy of inactivated or live-attenuated CAV-2 vaccines.

\section{Acknowledgments}

This study was supported financially by a grant (B1543083-2018-19-04) from the Animal and Plant Quarantine Agency, Ministry of Agriculture, Food and Rural Affairs (MAFRA), Republic of Korea.

\section{References}

1. Appel M, Carmichael LE, Robson DS. Canine adenovirus type 2-induced immunity to two canine adenoviruses in pubs with maternal antibody. Am J Vet Res 1975, 36, 1199-1202.

2. Balboni A, Dondi F, Prosperi S, Battilani M. Development of a SYBR Green real-time PCR assay with melting curve analysis for simultaneous detection and differentiation of canine adenovirus type 1 and type 2. J Virol Methods 2015, 222, 34-40.

3. Benetka V, Weissenböck H, Kudielka I, Pallan C, Rothmüller G, Möstl K. Canine adenovirus type 2 infection in four puppies with neurological signs. Vet Rec 2006, 158, 91-94.

4. Choi JW, Lee HK, Kim SH, Kim YH, Lee KK, Lee MH, Oem JK. Canine adenovirus type 1 in a fennec fox (Vulpes zerda). J Zoo Wildl Med 2014, 45, 947-950.

5. Goldstein T, Colegrove KM, Hanson M, Gulland FMD. Isolation of a novel adenovirus from California sea lions Zalophus californianus. Dis Aquat Organ 2011, 94, 243-248.

6. Gür S, Acar A. A retrospective investigation of canine adenovirus (CAV) infection in adult dogs in Turkey. J S Afr Vet Assoc 2009, 80, 84-86.

7. Kumar S, Stecher G, Tamura K. MEGA7: Molecular Evolutionary Genetics Analysis version 7.0 for bigger datasets. 
Mol Biol Evol 2016, 33, 1870-1874.

8. Lavan R, Knesl O. Prevalence of canine infectious respiratory pathogens in asymptomatic dogs presented at US animal shelters. J Small Anim Pract 2015, 56, 572-576.

9. Linné T. Differences in the E3 regions of the canine adenovirus type 1 and type 2. Virus Res 1992, 23, 119-133.

10. Loustalot F, Kremer EJ, Salinas S. The intracellular domain of the coxsackievirus and adenovirus receptor differentially influences adenovirus entry. J Virol 2015, 89, 9417-9426.

11. Millán J, López-Bao JV, García EJ, Oleaga A, Llaneza L, Palacios V, de la Torre A, Rodríguez A, Dubovi EJ, Esperón F. Patterns of exposure of Iberian wolves (Canis lupus) to canine viruses in human-dominated landscapes. Ecohealth 2016, 13, 123-134.

12. Mochizuki M, Hashimoto M, Ishida T. Recent epidemiological status of canine viral enteric infections and Giardia infection in Japan. J Vet Med Sci 2001, 63, 573-575.

13. Park NY, Lee MC, Kurkure NV, Cho HS. Canine adenovirus type 1 infection of a Eurasian river otter (Lutra lutra). Vet Pathol 2007, 44, 536-539.

14. Reed LJ, Muench H. Simple method of estimating fifty per cent endpoints. Am J Hyg 1938, 27, 493-497.
15. Sanjuán R, Donimgo-Calap P. Mechanisms of viral mutation. Cell Mol Life Sci 2016, 73, 4433-4448.

16. Szelechowski M, Fournier A, Richardson J, Eliot M, Klonjkowski B. Functional organization of the major late transcriptional unit of canine adenovirus type 2. J Gen Virol 2009, 90, 1215-1223.

17. Takamura K, Ajiki M, Hiramatsu K, Takemitsu S, Nakai M, Sasaki N. Isolation and properties of adenovirus from canine respiratory tract. Nihon Juigaku Zasshi 1982, 44, 355357.

18. Tham KM, Horner GW, Hunter R. Isolation and identification of canine adenovirus type -2 from the upper respiratory tract of a dog. N Z Vet J 1998, 46, 102-105.

19. Walker D, Abbondati E, Cox AL, Mitchell GBB, Pizzi R, Sharp CP, Philbey AW. Infectious canine hepatitis in red foxes (Vulpes vulpes) in wildlife rescue centres in the UK. Vet Rec 2016, 178, 421.

20. Yoon SS, Byun JW, Park YI, Kim MJ, Bae YC, Song JY. Comparison of the diagnostic methods on the canine adenovirus type 2 infection. Basic Appl Pathol 2010, 3, 5256. 